\title{
Efficacy and safety of a natural mineral water rich in magnesium and sulphate for bowel function: a double-blind, randomized, placebo-controlled study
}

\author{
Gordana Bothe $^{1} \cdot \mathrm{Aljaz}^{\mathrm{Coh}^{2}} \cdot$ Annegret Auinger ${ }^{1}$
}

Received: 25 June 2015 / Accepted: 27 October 2015 / Published online: 18 November 2015

(C) The Author(s) 2015. This article is published with open access at Springerlink.com

\begin{abstract}
Purpose The present placebo-controlled, double-blind, randomized trial aimed to investigate whether a natural mineral water rich in magnesium sulphate and sodium sulphate (Donat $\mathrm{Mg}$ ) may help to improve bowel function.

Methods A total of 106 otherwise healthy subjects with functional constipation were randomly assigned to consume 300 or $500 \mathrm{~mL}$ of a natural mineral water as compared to placebo water, over a course of 6 weeks. The 300$\mathrm{mL}$ arms were terminated due to the results of a planned interim analysis. Subjects documented the complete spontaneous bowel movements, spontaneous and overall bowel movements/week, stool consistency, gastrointestinal symptoms and general well-being in a diary. Change in the number of complete spontaneous bowel movements was defined as the primary outcome.

Results For the 75 subjects in the $500-\mathrm{mL}$ arms, the change in the number of complete spontaneous bowel movements per week tended to be higher in the active group when compared to placebo after 6 weeks $(T 2=1.8$; $p_{\text {value }}=0.036$; one-sided). The mean number of spontaneous bowel movements significantly increased over the course of the study, with significant differences between study arms considering the whole study time ( $F$ test $=4.743 ; p_{\text {time } \times \text { group }}=0.010,2$-sided). Stool consistency of spontaneous bowel movements $(p<0.001)$ and the subjectively perceived symptoms concerning constipation
\end{abstract}

Annegret Auinger

aauinger@a-r.com

1 Analyze \& Realize GmbH, Waldseeweg 6, 13467 Berlin, Germany

2 Droga Kolinska, d.d., Kolinska ulica 1, 1544 Ljubljana, Slovenia $(p=0.005)$ improved significantly with the natural mineral water as compared to placebo.

Conclusions The daily consumption of a natural mineral water rich in magnesium sulphate and sodium sulphate improved bowel movement frequency and stool consistency in subjects with functional constipation. Moreover, the subjects' health-related quality of life improved.

Clinical Trial Registration EudraCT No 2012-005130-11.

Keywords Bowel function - Stool frequency and consistency $\cdot$ Natural mineral water $\cdot$ Clinical trial

\section{Introduction}

Constipation is one of the leading bowel conditions affecting health-related quality of life but also comprising other complaints resulting in substantial healthcare costs [1-3]. Functional constipation is a functional gastrointestinal disorder characterized by straining during defecation, lumpy or hard stool, sensation of incomplete evacuation and infrequent bowel movements without evident organic or structural diseases [4]. An integrative review of eleven studies conducted in Asian, South American and European countries revealed a prevalence of constipation ranging from 2.6 to $26.9 \%$ in the general adult population [5]. In Europe, a mean prevalence rate of $17.1 \%$ was reported [6].

In principle, the spectrum of stool types in the general healthy population is wide including hard, fragmented lumps to sausage-like or snake-like forms up to mushy material, which correlates with transit time and faecal bulk [7]. The water content of stool usually ranges from 70 to $80 \%$, while constipated subjects have a harder stool with water content below $70 \%$ [8]. Stool consistency is frequently related to bowel movement, which also differs 
considerably between individuals, with a mean frequency of one stool per day reported for people living in Western countries [9].

Generally, the aetiology of constipation is very complex and mainly influenced by dietary factors including drinking habits and a sedentary lifestyle; hence, changes in lifestyle and diet are usually the first recommendation for constipated subjects [10]. The use of laxatives, bulking agents, stool softeners or other remedies is recommended if lifestyle modifications fail. These might, however, be associated with side effects; furthermore, according to a recent survey in ten European countries, $28 \%$ of subjects were dissatisfied with their current constipation treatment [10].

In a cross-sectional study on Japanese women, a low intake of magnesium and water from food was inversely associated with the prevalence of functional constipation. Indeed, magnesium salts such as magnesium sulphate are known for their osmotic effects accelerating intestinal transit time and leading to better stool consistency [11, 12]. Natural mineral waters rich in magnesium salts are therefore thought to improve bowel function. So far, controlled clinical trials assessing the effect of mineral-rich water on bowel function have not been frequently reported [13-15]. The aim of the present randomized, controlled interventional study was to examine the effect of a natural mineral water rich in magnesium sulphate and sodium sulphate as compared to placebo on bowel function in otherwise healthy adults with functional constipation.

\section{Methods}

\section{Trial design}

The study was designed as a mono-centre, parallel-treatment, multi-dose, double-blind study in subjects with functional constipation. The trial incorporated a planned, adaptive interim analysis to adjust for sample size and to evaluate the different doses with regard to the main study objective. The first part of the study was performed with four study arms: two arms with a daily dose of $300 \mathrm{~mL}$ (active and placebo) and two arms with a daily dose of $500 \mathrm{~mL}$ (active and placebo). Following the interim analysis, the lower-volume/lower-dose arms were terminated, and the final sample size (for the $500 \mathrm{~mL}$ dose) was adjusted. Results for the lower dose are not presented.

The study was approved by the Ethics Committee (Office for Health and Social Affairs, Berlin, Germany) and by the Competent Authority (Federal Institute for Drugs and Medical Devices, Bonn, Germany). It was conducted in compliance with the Declaration of Helsinki as well as the German Pharmaceuticals Act, the principles of ICH-GCP, and the German GCP-V. The study was registered in the
European Clinical Trials Database (EudraCT) as EudraCT No 2012-005130-11. Participants gave written informed consent prior to the study.

\section{Subjects}

A total of 106 otherwise healthy subjects with functional constipation were enrolled to the study site in Berlin, Germany. Subjects aged 18-70 with functional constipation according to ROME III criteria having two-to-four bowel movements per week during the preceding months were included $[4,16]$. They were asked to adhere to their former diet and physical activity and had to be used to consuming at least $300 \mathrm{~mL}$ of water (incl. tea). Women of childbearing potential had to agree to use contraception methods. The exclusion criteria were as follows: acute gastritis and enteritis, bleeding tendency and risk of rupture in the intestinal tract, disorders in motility and secretion in the digestive tract, acute or chronic disease of the gastrointestinal tract, irritable bowel syndrome, abdominal pain, abdominal surgery within the last 6 months prior to study start, known pelvic floor dysfunction, susceptibility to development of kidney stones, hyperresorptive hypercalciuria with urinary stones, urinary infections with $E$. coli, renal insufficiency, acute or chronic kidney or urinary tract disease, alkalosis, severe respiratory disease, cardiovascular system insufficiency, acute inflammatory diseases, dehydrated conditions, restricted fluid tolerance, acute or chronic neurological or psychiatric illness, weight loss of $\geq 3 \mathrm{~kg}$ within the last 3 months prior to the study, clinically relevant excursions of laboratory parameters, BMI $>35 \mathrm{~kg} /$ $\mathrm{m} 2$, thyroid dysfunction, known sensitivity to the ingredients of the product, use of any preparations that could affect the gastrointestinal tract during the last 2 weeks and during the study (except rescue medication, a bisacodyl suppository that could be used in case of no bowel movements for 4 days; max. four suppositories were permitted during the entire study), use of sympathomimetics and cardiac glycosides, supplementation of magnesium, vitamins or other minerals during the study, intake of mineral water other than the study product during the study, pregnancy or nursing, drug, alcohol or medication abuse, participation in another clinical trial during the last 30 days prior to study start, relationship with or dependence on the sponsor or the investigator, problems with complying with or following the protocol due to language difficulties, or commitment to an institution by virtue of an order issued either by the judicial or the administrative authorities.

Furthermore, subjects had to fulfil the following randomisation criteria during the run-in period before obtaining the investigational product (assessed per subject diary): two-to-four bowel movements per week and intake of at least $300 \mathrm{~mL}$ water (incl. tea) daily. 


\section{Intervention}

During the study period of 6 weeks, the subjects consumed their daily dose of Donat Mg natural mineral water or placebo in two portions: prior to breakfast and in the evening before dinner. Donat $\mathrm{Mg}$ natural mineral water is derived from a spring in Rogaska Slatina, Slovenia. It is enriched by minerals from dissolving rocks $280-600 \mathrm{~m}$ under ground providing Donat $\mathrm{Mg}$ with $13 \mathrm{~g} / \mathrm{L}$ of dissolved mineral substances. Both the mineral water and the placebo were produced and bottled by Droga Kolinska, d.d (Slovenia). Main ingredients of Donat $\mathrm{Mg}$ natural mineral water are sodium $(1600 \mathrm{mg} / \mathrm{L})$, magnesium $(1000 \mathrm{mg} / \mathrm{L})$, calcium $(370 \mathrm{mg} / \mathrm{L})$, sulphate $(2000 \mathrm{mg} / \mathrm{L})$, and hydrogen carbonate $(7600 \mathrm{mg} / \mathrm{L})$. The placebo was sparkling water derived from another spring in Rogaska Slatina with a low content of minerals $(<1 \mathrm{mg} / \mathrm{L}$ sodium, $30 \mathrm{mg} / \mathrm{L}$ magnesium, $73 \mathrm{mg} / \mathrm{L}$ calcium, $17 \mathrm{mg} / \mathrm{L}$ sulphate, $390 \mathrm{mg} / \mathrm{L}$ hydrogen carbonate) and a quantity of $\mathrm{CO}_{2}(3.5 \mathrm{~g} / \mathrm{L})$ comparable to the active product.

The clinical phase of the study included a run-in period of $10 \pm 2$ days and an intervention period of 6 weeks \pm 3 days. A total of five visits were performed: a screening visit, a baseline visit (after the run-in period), a telephone visit $7 \pm 3$ days after baseline, a control visit $21 \pm 3$ days after baseline and the final visit $42 \pm 3$ days after baseline.

Compliance was checked by counting returned unused investigational product and assessing the trial duration. The accepted compliance rate was defined as $75-125 \%$ of the correct quantity of investigational product and max. 3 days deviation from the 6-week study period.

\section{Outcome measures}

The primary outcome was the subject-rated change in the number of complete spontaneous bowel movements (CSBMs) per week between placebo and active over the course of the study. A CSBM was defined as a bowel movement with sensation of complete evacuation and with no laxative/enema in the $24 \mathrm{~h}$ preceding the bowel movement. In addition, the number of overall bowel movements (BM), the number of spontaneous bowel movements (SBM, bowel movements with no laxative/enema in the $24 \mathrm{~h}$ preceding the bowel movement) and the number of complete bowel movements (CBM, defined as bowel movements with a sensation of complete evacuation) were assessed. Subjects were asked to record their stool consistency using Bristol Stool Form Scale (BSFS) questionnaire [17].

Subjects had to document their daily bowel movements, the respective stool form, the sensation of complete evacuation following defecation (yes/no) and whether they had used any rescue medication in a daily diary. In addition, subjects had to fill in the questionnaires Gastrointestinal Symptoms Rating Scale (GSRS) [18], the Short Form 12 Health Survey Questionnaire (SF-12) [19] and the short form of the international physical activity questionnaire (IPAQ-SF) at each visit post screening [20]. Eating and drinking habits were recorded in a diary on 3 days of each week (to calculate the mean answer to the items regarding intake of certain foods/day, the following score was used: none $=1$, once $=2$, twice or three times $=3$, more than three times $=4$; the intake of liquids was recorded in $\mathrm{mL}$ ). The efficacy of the investigational products was evaluated by the participants and the investigator independently at the end of the study by means of a global-scaled evaluation with "very good", "good", "moderate" or "poor".

Biochemical parameters such as liver function and lipid parameters as well as blood pressure and heart rate were assessed at screening and at the end of the intervention.

\section{Adaptive design and statistics}

The adaptive interim analysis was scheduled to take place when data from $50 \%$ of originally planned number of subjects were available [21]. The analysis was done by an independent statistician using the full analysis set (FAS). An Independent Data Monitoring Committee (IDMC) was in place to provide appropriate recommendations to the trial sponsor while all staff involved in the conduct of the trial remained blind to the results of the interim analysis. Two sets of one-sided confirmatory hypotheses were planned to be tested at the interim and final analysis for the primary efficacy variable dCSBM in each of the dose groups (i.e. $300 \mathrm{~mL}$ each verum or placebo; $500 \mathrm{~mL}$ each verum or placebo) (Table 1).

The trial results were analysed according to a two-stage adaptive group sequential design with one interim analysis using O'Brien and Fleming stopping boundaries [21]. Each hypothesis was tested (at the interim and final analysis)
Table 1 Splitting of the overall type I error

\begin{tabular}{|c|c|c|c|c|c|c|}
\hline \multirow[t]{3}{*}{ Alpha (one-sided) } & \multirow[t]{3}{*}{ Stage } & \multirow[t]{3}{*}{ Analysis } & \multicolumn{4}{|c|}{ Critical value to reject $\mathrm{H} 0$} \\
\hline & & & \multicolumn{2}{|l|}{$\mathrm{H}(01)$} & \multicolumn{2}{|l|}{$\mathrm{H}(02)$} \\
\hline & & & Test statistic & Alpha (one-sided) & Test statistic & Alpha (one-sided) \\
\hline \multirow[t]{2}{*}{0.025} & 1 & Interim & 3.011 & 0.0013 & 2.797 & 0.0026 \\
\hline & 2 & Final & 2.257 & 0.0120 & 1.977 & 0.0240 \\
\hline
\end{tabular}


with the nonparametric one-sided Mann-Whitney $U$ test. The inverse-normal method was used to combine the $p$ values for each of the two sets of one-sided confirmatory hypotheses [22].

The overall experiment-wise significance level [i.e. the family-wise error rate (FWER)] was set to $=0.025$ (onesided). Adjustment of the significance level was performed to control the overall type I error $=0.025$ (one-sided). In the interim analysis, the null hypotheses could not be rejected for any of the dose levels. The IDMC recommended stopping the low-volume-dose groups and to adjust the sample size for the higher dose. The final analysis of the primary endpoint was only performed for the 500-mL dose with the test statistic $T 2$ critical value to reject the null hypothesis of 1.977 . The secondary outcome parameters were tested in the exploratory sense using the nonparametric procedures Mann-Whitney $U$ test, Wilcoxon test and Chi-square test. Changes in clinical parameters over time were analysed by using exploratory ANOVA. The analysis was performed using the FAS and at least for the primary outcome in addition using the valid case analysis set (VCAS).

\section{Sample size estimation}

For the sample size estimation before the interim analysis, the calculation was based on the assumption of an effect size of 0.7 for the lower dose and 1.0 for the higher dose. The sample size re-estimation for the study continuation with the higher dose was calculated based on the target conditional power using the procedure suggested by Chang [23]. The initial estimates and the observed treatment difference and standard deviation at the interim analysis were considered. The conditional power was set to $80 \%$. Critical value $\alpha 2$ for the final analysis was set to 0.0240 .

\section{Results}

\section{Subject recruitment}

Out of the 132 subjects assessed for eligibility, 106 were randomized (Fig. 1); 30 subjects thereof were allocated to the $300-\mathrm{mL}$ arms, which were terminated after the interim analysis (data not shown). Of the 76 subjects allocated to the $500-\mathrm{mL}$ arms, 75 were included in the FAS population (one subject had no data except baseline).

\section{Characteristics of study subjects at baseline}

Of the 75 subjects in the 500-mL arms, 63 were female and 12 were male with functional constipation according to ROME III (Table 2). The baseline characteristics, including the eating and drinking habits and physical activity did not differ between interventional groups.

\section{Bowel movements}

All bowel movements analysed were spontaneous; hence, the results given for CSBMs are also valid for CBM assessment and those for SBMs accordingly for BM assessment. The change in the number of complete spontaneous bowel movements per week tended to be higher in the active group as compared to placebo after 6 weeks (test statistic $T 2=1.8$; combined $p_{\text {value }}=0.036$ with a confirmatory significance level of 0.024 ; one-sided). There was a trend between the active group and the placebo group regarding the changes in CSBMs over time ( $F$ test: 2.992; $p_{\text {time } \times \text { group }}=0.054$; Table 3 ).

The mean number of SBMs/BMs almost doubled after 6 weeks of drinking the mineral-rich water, with significant differences between study arms considering the whole study time $\left(F\right.$ test $=4.743 ; p_{\text {time } \times \text { group }}=0.010$, Table 3$)$. After three and 6 weeks, respectively, the number in SBMs/ BMs was significantly higher in subjects drinking the mineral-rich water as compared to those drinking placebo water ( $p=0.006$ and $p=0.001$, respectively).

Similar results were shown for VCAS; there were no statistically significant differences between the active and placebo groups in changes in CSBMs per week $(p=0.154)$, while for the SBMs/BMs the groups differed significantly $(p=0.024)$.

\section{Stool consistency}

With regards to the consistency of SBMs/BMs, $78.4 \%$ in the active group and $60.5 \%$ in the placebo group reported that the stool became softer after 6 weeks of intake, while $8.1 \%$ and $23.7 \%$ in the corresponding groups showed a harder stool as compared to baseline ( $p_{\text {active }}<0.001$; $p_{\text {placebo }}=0.012$ ). At week 3 and 6 , subjects in the active group had a significant softer stool than the placebo group ( $p=0.001$ and $p<0.001$, respectively; Fig. 2). The stool consistency of SBMs/BMs throughout the study differed significantly between study groups $(F$ test $=12.376$; $\left.p_{\text {time } \times \text { group }}<0.001\right)$.

\section{Gastrointestinal symptoms and general well-being}

The gastrointestinal symptoms were assessed by means of the GSRS. In line with the increased number of bowel movements and stool consistency score, subjects drinking the mineral-rich water experienced an improvement in the symptoms related to constipation throughout the study $(p=0.005)$, while other symptom clusters and the total sum score did not differ between study arms (Table 4). 


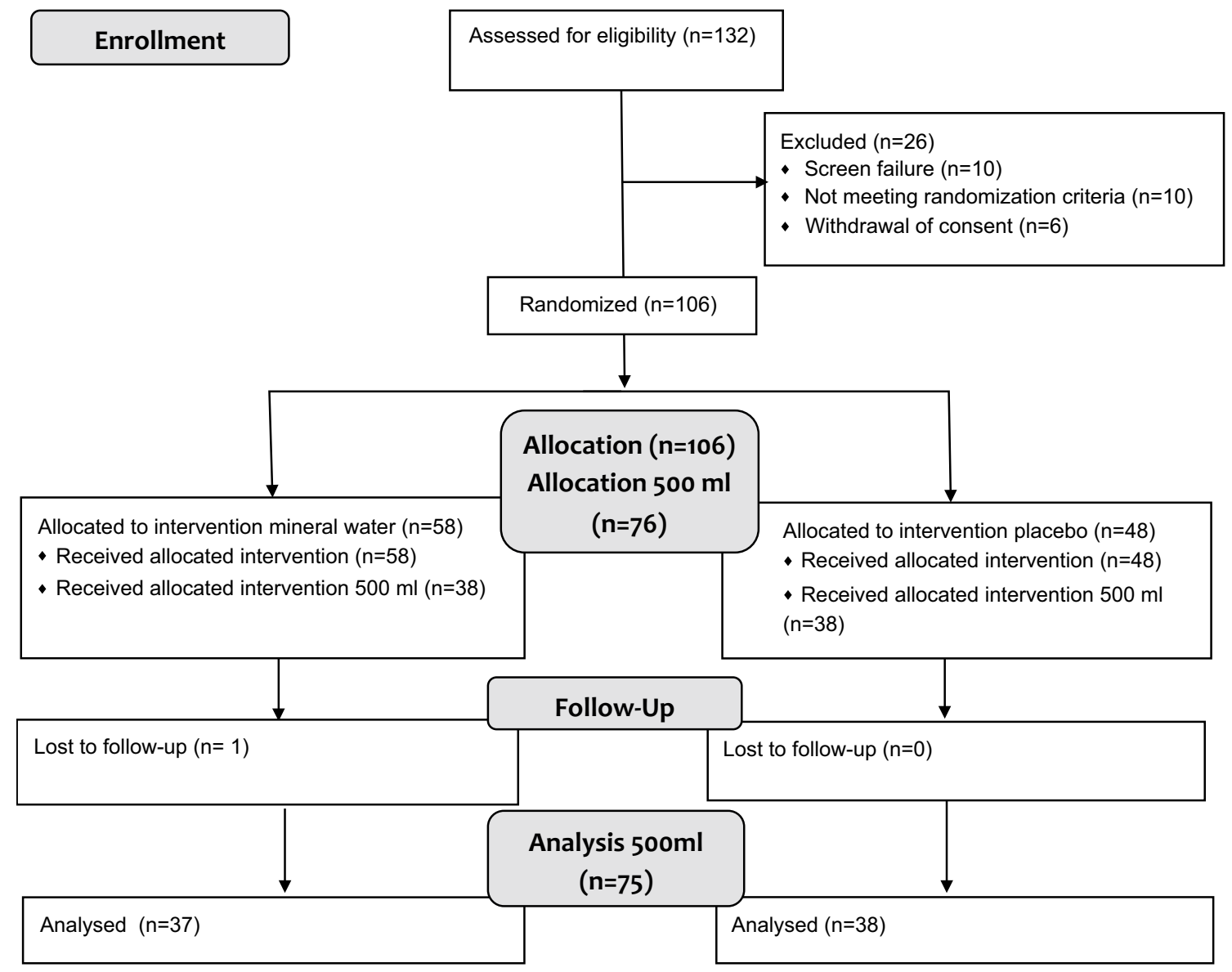

Fig. 1 Subject flow chart

Table 2 Baseline characteristics of study subjects

\begin{tabular}{lllll}
\hline & Total & Active & Placebo & $p$ \\
\hline Gender (f/m) & $63 / 12$ & $31 / 6$ & $32 / 6$ & 0.960 \\
Age & $46.4 \pm 12.6$ & $46.8 \pm 12.9$ & $46.0 \pm 12.5$ & 0.707 \\
Weight (cm) & $70.6 \pm 11.9$ & $71.1 \pm 13.6$ & $70.2 \pm 10.2$ & 0.899 \\
Systolic blood pressure (mmHg) & $115.2 \pm 12.1$ & $113.9 \pm 11.3$ & $116.5 \pm 12.9$ & 0.332 \\
Diastolic blood pressure (mmHg) & $75.4 \pm 6.6$ & $74.9 \pm 6.2$ & $75.9 \pm 7.0$ & 0.419 \\
\hline
\end{tabular}

$p$ values (Mann-Whitney $U$ test) for differences between study arms
In addition, there were significant differences between the mineral-rich water group and the placebo group with regards to changes in the SF-12 sum score from baseline to week $6(p=0.017)$.

\section{Physical performance and dietary habits}

Physical activity was not changed in any of the study groups over the course of the study $(p=0.628)$. There was no significant difference between study groups in the consumption of beverages throughout the entire study. At the end of the intervention, the active group ate slightly more dried fruits (from 1.04 at baseline to 1.15 at 6 weeks) and carrots/potatoes (from 1.53 at baseline to 1.68 at 6 weeks) as compared to placebo (1.04 at baseline to 1.03 at 6 weeks for dried fruits, $p=0.04 ; 1.68$ at baseline to 1.60 at 6 weeks for carrots/potatoes; $p=0.03$ ).

\section{Global evaluation of the efficacy}

At the end of the study, $94.5 \%$ of subjects in the mineral-rich water group-compared to $57.9 \%$ in the placebo group-rated the global evaluation of efficacy as "very good" or "good". Similarly, the physicians rated the 
Table 3 Mean (SD) number of CSBMs per week and SBMs/BMs per week during a 6-week intervention with mineral-rich water or placebo water

\begin{tabular}{|c|c|c|c|c|c|}
\hline & \multicolumn{2}{|l|}{ Active } & \multicolumn{2}{|c|}{ Placebo } & \multirow[t]{2}{*}{$p_{\mathrm{U}}$} \\
\hline & Mean & SD & Mean & SD & \\
\hline \multicolumn{6}{|c|}{ CSBMs per week } \\
\hline Baseline & 0.68 & $(1.11)$ & 0.79 & $(1.10)$ & 0.629 \\
\hline Week 3 & 2.19 & (3.19) & 1.26 & $(1.64)$ & 0.353 \\
\hline Week 6 & 2.14 & $(2.67)$ & 1.16 & $(1.50)$ & 0.173 \\
\hline$p_{\text {ANOVA }}$ & 0.054 & & & & \\
\hline \multicolumn{6}{|c|}{ SBMs/BMs per week } \\
\hline Baseline & 3.38 & $(1.26)$ & 3.03 & $(0.92)$ & 0.329 \\
\hline Week 3 & 6.14 & $(3.32)$ & 4.45 & $(2.09)$ & 0.006 \\
\hline Week 6 & 6.62 & $(3.20)$ & 4.47 & $(2.20)$ & 0.001 \\
\hline$p_{\text {ANOVA }}$ & 0.010 & & & & \\
\hline
\end{tabular}

$p_{\mathrm{U}}$ values (Mann-Whitney $U$ test) for differences between study arms $p_{\text {ANOVA }}$ for RM-ANOVA (time $\times$ group interaction)

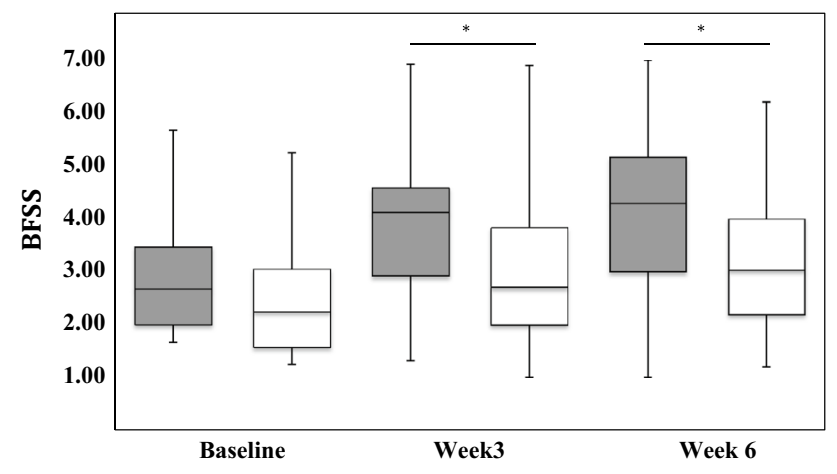

Fig. 2 Stool consistency SBM/BM using bristol stool form scale (BSFS type, median, interquartile range, range) over the course of the intervention (grey active group, white placebo group). Bristol stool score -1 for hard lumps to seven watery stools. * $p$ values (MannWhitney $U$ test) for differences between study arms

mineral-rich water as "very good" or "good" for $97.2 \%$ of the participants, while they gave the same rating for $57.9 \%$ of the participants consuming the placebo water. Both participants and physicians rated the efficacy of the mineralrich water better than the placebo water $\left(p_{\text {chi }}=0.001\right.$ and $p_{\text {chi }}<0.001$, respectively).

\section{Safety evaluation, laboratory parameters and use of rescue medication}

A total of 16 adverse events occurred during the study period in the $500-\mathrm{mL}$ arm with no statistical difference between the mineral water group and placebo group $(p=0.571)$. Twelve occurred in the active group, and another four events were recorded in the placebo
Table 4 Mean (SD) of gastrointestinal symptom rating scale (GSRS)

\begin{tabular}{|c|c|c|c|c|c|}
\hline & \multicolumn{2}{|l|}{ Active } & \multicolumn{2}{|c|}{ Placebo } & \multirow[t]{2}{*}{$p$} \\
\hline & Mean & SD & Mean & SD & \\
\hline \multicolumn{6}{|c|}{ GSRS (reflux) } \\
\hline Baseline & 1.54 & 0.96 & 1.37 & 0.72 & 0.413 \\
\hline Week 6 & 1.39 & 0.63 & 1.45 & 0.79 & 0.990 \\
\hline$p_{\mathrm{U} \Delta}$ & 0.348 & & & & \\
\hline \multicolumn{6}{|c|}{ GSRS (abdominal pain) } \\
\hline Baseline & 1.90 & 0.74 & 1.96 & 0.80 & 0.797 \\
\hline Week 6 & 1.66 & 0.74 & 1.68 & 0.72 & 0.773 \\
\hline$p_{\mathrm{U} \Delta}$ & 0.944 & & & & \\
\hline \multicolumn{6}{|c|}{ GSRS (indigestion) } \\
\hline Baseline & 2.75 & 1.19 & 2.72 & 1.02 & 0.924 \\
\hline Week 6 & 2.26 & 1.13 & 2.30 & 0.99 & 0.617 \\
\hline$p_{\mathrm{U} \Delta}$ & 0.915 & & & & \\
\hline \multicolumn{6}{|c|}{ GSRS (diarrhoea) } \\
\hline Baseline & 1.23 & 0.49 & 1.23 & 0.56 & 0.492 \\
\hline Week 6 & 1.64 & 1.04 & 1.35 & 0.55 & 0.392 \\
\hline$p_{\mathrm{U} \Delta}$ & 0.335 & & & & \\
\hline \multicolumn{6}{|c|}{ GSRS (constipation) } \\
\hline Baseline & 3.82 & 1.48 & 3.59 & 1.38 & 0.419 \\
\hline Week 6 & 1.81 & 0.79 & 2.56 & 1.14 & 0.001 \\
\hline$p_{\mathrm{U} \Delta}$ & 0.005 & & & & \\
\hline \multicolumn{6}{|c|}{ GSRS (total) } \\
\hline Baseline & 2.33 & 0.76 & 2.26 & 0.70 & 0.722 \\
\hline Week 6 & 1.81 & 0.58 & 1.92 & 0.66 & 0.442 \\
\hline$p_{\mathrm{U} \Delta}$ & 0.327 & & & & \\
\hline
\end{tabular}

$p_{\mathrm{U} \Delta}$ values (Mann-Whitney $U$ test) for differences between study arms in changes in GSRS dimension between baseline and final visit $p$ values (Mann-Whitney $U$ test) for differences between study arms

group. One case in the active group might be related to the intervention. In two cases of the active group, the causality of the adverse event was probably related to the intervention. One adverse event in the active group was classified as serious but was not related to the intervention.

None of the measured clinical parameters including liver enzymes, triglycerides, cholesterol, HDL-C, LDL-C, blood pressure and heart rate values differed between study groups.

With regards to rescue medication, no difference was observed between groups regarding the intake of rescue medication $(p=0.240)$.

There were no statistically significant differences between groups in deviation of the actual intake of investigational products from expected intake $(p>0.1)$. The compliance rate considering the intake of the investigational product was $102 \% \pm 8.6$ in the active group and $105 \% \pm 9.4$ in the placebo group. 


\section{Discussion}

The present placebo-controlled, randomized, double-blind intervention study in subjects with functional constipation provides clinical evidence that drinking a mineral water rich in magnesium sulphate and sodium sulphate over a course of 6 weeks can confer significant benefits for digestive health. The improvement in the constipation symptoms as reflected by an increased number of CSBMs and overall bowel movements per week and a softer stool in comparison with a water low in minerals confirmed previous assumptions of the digestion stimulating effects of waters naturally rich in minerals. Indeed, the number of CSBMs/ week more than tripled within the course of the study in subjects drinking the mineral-rich water, which is comparable to the effect of psyllium and dried plums demonstrating an increase in the mean number of CSBMs/week by more than a double [24].

In line with the improved objective parameters of bowel function, study participants experienced an improvement in the GSRS dimension constipation exceeding the minimal clinically relevant score change of 0.5 [25] together with an overall increase in the health-related quality of life.

The change in the number of CSBMs per week was chosen as the primary endpoint as it is considered the more sensitive assessment with regards to the overall bowel function as information on the completeness of bowel movement is also provided [26]. Since there was no adequate and well-controlled trial with the investigational product available at the time the current study was planned, an adaptive study design was applied to adjust the sample size, if necessary or to terminate the entire trial, the low- (300$\mathrm{mL}$ ) or the high- $(500-\mathrm{mL})$ volume dose arms earlier if efficacy in the primary endpoint is lacking or already achieved at the interim analysis. In terms of CSBMs, participants did not seem to benefit from drinking $300 \mathrm{~mL}$ of the mineral water per day, and therefore these arms of the study were closed, while the sample size of the 500-mL dose group was increased based on the results of the interim analysis. Given the outcomes of this clinical trial, the intake of $500 \mathrm{~mL}$ of Donat $\mathrm{Mg}$ per day seems to improve CSBMs but also overall bowel movements and consistency more efficiently than the 300-mL dose.

As dietary habits and lifestyle factors are thought to impact bowel function, the subjects' eating and drinking habits and physical activity levels were assessed. Only a marginal increase in the consumption of dried fruits was observed in the active group as compared to placebo over the course of the study. Given the semi-quantitative nature of the dietary assessment and the observed small differences between study groups, it is highly unlikely that this change might have had an impact on the study results.
The outcome of the present clinical trial is in agreement with another recently published study comparing different dosages of a mineral water with placebo. In that trial, the daily consumption of $1 \mathrm{~L}$ magnesium sulphate-rich mineral water (119 mg/L magnesium, $1530 \mathrm{mg} / \mathrm{L}$ sulphate) reduced constipation and improved stool consistency in functionally constipated women [13]. Although the study group was limited to women and the primary endpoint was response to the treatment as assessed by components of the Rome III criteria, while the number of bowel movements was not reported, this study confirms the beneficial effect of a natural mineral water rich in magnesium and sulphate on bowel function.

In the present study, a mineral water containing $13 \mathrm{~g} / \mathrm{L}$ of dissolved mineral substances, among them magnesium sulphate and sodium sulphate was investigated. Magnesium sulphate exerts its osmotic effect by trapping water in the intestinal lumen, resulting in increased and softer faecal bulk, which presses on the intestinal wall provoking peristalsis [27, 28]. A study in healthy human subjects demonstrated that high doses of orally administered magnesium sulphate accelerate small intestinal transit time and modulate antroduodenal motility in the fasting but not in the postprandial state [11]. More recently, in vivo and in vitro studies showed that, along with the magnesium sulphateinduced change in the osmotic pressure, the expression of osmoregulatory genes is induced $[29,30]$. In addition, the expression of Aquaporin 3, a gene involved in the regulation of faecal water content in the colon, was increased in the rat colon after administration of magnesium sulphate [30]. Another study in rats reported that the laxative effect of magnesium sulphate may involve the release of nitric oxide (NO) through the stimulation of NO synthase [31]. Sulphate is further known to increase faecal bulk and stool consistency, and water containing more than $1000 \mathrm{mg} / \mathrm{L}$ sulphate was linked to a self-reported laxative effect [32, 33]. Overall, the effect on stool frequency and consistency of the investigated mineral water is most likely related to its naturally high content of magnesium and sulphate.

Some limitations of the study should be noted. In addition to the effect of the mineral water on constipation, its influence on the entire gastrointestinal tract and the overall quality of life was evaluated. However, it might have been more appropriate to use constipation-specific questionnaires to assess the respective symptoms and quality of life rather than the more general tools applied in the study. Further, after $50 \%$ of the originally planned number of subjects have been recruited, the lower-dose group was stopped based on the recommendation made by the IDMC. Yet, the sample size might have been too small to draw a firm conclusion for the 300-mL group. Further clinical trials with larger sample size might investigate an effect of the mineral-rich water. In addition, measuring other gut 
health-related parameters such as faecal bulk, transit time or a potential impact on microbiota would further elucidate the underlying mode of action.

In summary, the presented study in otherwise healthy subjects confirms the beneficial effect of drinking $500 \mathrm{~mL}$ natural mineral water daily on bowel function in subjects with functional constipation. In addition, the consumption of this specific mineral water was proven to be safe and tolerable. The observed results support the postulation that a mineral water naturally rich in magnesium sulphate and sodium sulphate may be considered as a first line of recommendation for subjects with less frequent bowel movements or harder stool in order to maintain a normal defecation. This in turn may help to reduce gastrointestinal discomfort and the development of diseases frequently associated with constipation. Finally, this might improve the health-related quality of life and subsequently reduce the economic burden on healthcare resources.

Acknowledgments We would like to thank Norman Bitterlich, Ph.D. (Medizin \& Service GmbH) and Werner Baurecht (acromion $\mathrm{GmbH}$ ) for their crucial support in statistical analysis of the data.

\section{Compliance with ethical standards}

Conflict of interest The study was funded by Droga Kolinska, d.d (Ljubljana, Slovenia). Aljaz Coh is employed by Droga Kolinska, d.d Gordana Bothe and Annegret Auinger are employed by a contract research organization. The authors declare no other competing interests regarding this study.

Open Access This article is distributed under the terms of the Creative Commons Attribution 4.0 International License (http://creativecommons.org/licenses/by/4.0/), which permits unrestricted use, distribution, and reproduction in any medium, provided you give appropriate credit to the original author(s) and the source, provide a link to the Creative Commons license, and indicate if changes were made.

\section{References}

1. Norton C (2006) Constipation in older patients: effects on quality of life. Br J Nurs. doi:10.12968/bjon.2006.15.4.20542

2. Burkitt DP, Walker AR, Painter NS (1972) Effect of dietary fibre on stools and the transit-times, and its role in the causation of disease. Lancet 2(7792):1408-1412

3. Sun SX, Dibonaventura M, Purayidathil FW, Wagner JS, Dabbous O, Mody R (2011) Impact of chronic constipation on health-related quality of life, work productivity, and healthcare resource use: an analysis of the National Health and Wellness Survey. Dig Dis Sci. doi:10.1007/s10620-011-1639-5

4. Drossman DA (2006) The functional gastrointestinal disorders and the Rome III process. Gastroenterology. doi:10.1053/j. gastro.2006.03.008

5. Schmidt FM, Santos VL (2014) Prevalence of constipation in the general adult population: an integrative review. J Wound Ostomy Cont Nurs. doi:10.1097/01.WON.0000438019.21229.b7
6. Peppas G, Alexiou VG, Mourtzoukou E, Falagas ME (2008) Epidemiology of constipation in Europe and Oceania: a systematic review. BMC Gastroenterol. doi:10.1186/1471-230X-8-5

7. Wyman JB, Heaton KW, Manning AP, Wicks AC (1978) Variability of colonic function in healthy subjects. Gut 19(2):146-150

8. Wenzl HH, Fine KD, Schiller LR, Fordtran JS (1995) Determinants of decreased fecal consistency in patients with diarrhea. Gastroenterology 108(6):1729-1738

9. Weaver LT (1988) Bowel habit from birth to old age. J Pediatr Gastroenterol Nutr 7(5):637-640

10. Muller-Lissner S, Tack J, Feng Y, Schenck F, Specht Gryp R (2013) Levels of satisfaction with current chronic constipation treatment options in Europe-an internet survey. Aliment Pharmacol Ther. doi:10.1111/apt.12124

11. Vu MK, Nouwens MA, Biemond I, Lamers CB, Masclee AA (2000) The osmotic laxative magnesium sulphate activates the ileal brake. Aliment Pharmacol Ther 14(5):587-595

12. Stewart JJ, Gaginella TS, Olsen WA, Bass P (1975) Inhibitory actions of laxatives on motility and water and electrolyte transport in the gastrointestinal tract. J Pharmacol Exp Ther 192(2):458-467

13. Dupont C, Campagne A, Constant F (2014) Efficacy and safety of a magnesium sulfate-rich natural mineral water for patients with functional constipation. Clin Gastroenterol Hepatol. doi:10.1016/j.cgh.2013.12.005

14. Constant F, Morali A, Arnaud MJ, Delabroise AM, Thirion F, Matisse N, Wagner M, Dohm JP, Vuillemin JL, Gay G (1999) Treatment of idiopathic constipation in infants: comparative and randomized study of twa mineral waters (60 cases). J Pediatr Gastroenterol Nutr 28(5):551

15. Gasbarrini G, Candelli M, Graziosetto RG, Coccheri S, Di Iorio F, Nappi G (2006) Evaluation of thermal water in patients with functional dyspepsia and irritable bowel syndrome accompanying constipation. World J Gastroenterol 12(16):2556-2562

16. Drossman DA (2006) Rome III: the new criteria. Chin J Dig Dis. doi:10.1111/j.1443-9573.2006.00265.x

17. Lewis SJ, Heaton KW (1997) Stool form scale as a useful guide to intestinal transit time. Scand J Gastroenterol. doi:10.3109/00365529709011203

18. Dimenas E, Glise H, Hallerback B, Hernqvist H, Svedlund J, Wiklund I (1995) Well-being and gastrointestinal symptoms among patients referred to endoscopy owing to suspected duodenal ulcer. Scand J Gastroenterol 30(11):1046-1052

19. Ware J Jr, Kosinski M, Keller SD (1996) A 12-Item Short-Form Health Survey: construction of scales and preliminary tests of reliability and validity. Med Care 34(3):220-233

20. Lee PH, Macfarlane DJ, Lam TH, Stewart SM (2011) Validity of the international physical activity questionnaire short form (IPAQ-SF): a systematic review. Int J Behav Nutr Phys Act. doi:10.1186/1479-5868-8-115

21. Chow S-C, Chang M (2012) Adaptive design methods in clinical trials. Chapman \& Hall/CRC, New York

22. Lehmacher W, Wassmer G (1999) Adaptive sample size calculations in group sequential trials. Biometrics 55(4):1286-1290

23. Chang M (2008) Adaptive design theory and implementation using SAS and R. Chapman \& Hall/CRC, New York

24. Attaluri A, Donahoe R, Valestin J, Brown K, Rao SS (2011) Randomised clinical trial: dried plums (prunes) vs. psyllium for constipation. Aliment Pharmacol Ther. doi:10.1111/j.1365-2036.2011.04594.x

25. Talley NJ, Fullerton S, Junghard O, Wiklund I (2001) Quality of life in patients with endoscopy-negative heartburn: reliability and sensitivity of disease-specific instruments. Am J Gastroenterol. doi:10.1111/j.1572-0241.2001.03932.x

26. Mueller-Lissner S, Kamm MA, Wald A, Hinkel U, Koehler U, Richter E, Bubeck J (2010) Multicenter, 4-week, double-blind, 
randomized, placebo-controlled trial of sodium picosulfate in patients with chronic constipation. Am J Gastroenterol. doi:10.1038/ajg.2010.41

27. Donowitz M (1979) Current concepts of laxative action: mechanisms by which laxatives increase stool water. J Clin Gastroenterol 1(1):77-84

28. Izzo AA, Gaginella TS, Capasso F (1996) The osmotic and intrinsic mechanisms of the pharmacological laxative action of oral high doses of magnesium sulphate. Importance of the release of digestive polypeptides and nitric oxide. Magnes Res 9(2):133-138

29. Ikarashi N, Mochiduki T, Takasaki A, Ushiki T, Baba K, Ishii M, Kudo T, Ito K, Toda T, Ochiai W, Sugiyama K (2011) A mechanism by which the osmotic laxative magnesium sulphate increases the intestinal aquaporin 3 expression in HT-29 cells. Life Sci. doi:10.1016/j.lfs.2010.11.013
30. Ikarashi N, Ushiki T, Mochizuki T, Toda T, Kudo T, Baba K, Ishii M, Ito K, Ochiai W, Sugiyama K (2011) Effects of magnesium sulphate administration on aquaporin 3 in rat gastrointestinal tract. Biol Pharm Bull 34(2):238-242

31. Izzo AA, Gaginella TS, Mascolo N, Capasso F (1994) Nitric oxide as a mediator of the laxative action of magnesium sulphate. Br J Pharmacol 113(1):228-232

32. Moore EW (1952) Physiological effects of the consumption of saline drinking water. In: A progress report to the 16th meeting of the subcommittee on sanitary engineering and environment. Appendix B. National Academy of Sciences, Washington, DC

33. Heizer WD, Sandler RS, Seal E Jr, Murray SC, Busby MG, Schliebe BG, Pusek SN (1997) Intestinal effects of sulfate in drinking water on normal human subjects. Dig Dis Sci 42(5):1055-1061 